\title{
Inhibition of Methylglyoxal-Induced AGEs/ RAGE Expression Contributes to Dermal Protection by N-Acetyl-L-Cysteine
}

\author{
Chun-tao Yang ${ }^{\mathrm{a}, \mathrm{b}}$ Fu-hui Meng ${ }^{\mathrm{a}}$ Li Chen $^{\mathrm{a}} \quad$ Xiang Li $^{\mathrm{a}}$ Lai-Jian Cen $^{\mathrm{a}}$ Yu-hua Wen ${ }^{\mathrm{a}}$ \\ Cai-chen Lia Hui Zhang ${ }^{a, b, c}$ \\ aKey Laboratory of Protein Modification and Degradation, School of Basic Medical Sciences, ${ }^{b}$ Affiliated \\ Cancer Hospital \& Institute, CQuality Control Section of Academic Affairs, Guangzhou Medical \\ University, Guangzhou, China
}

\section{Key Words}

Advanced glycation end products • Delayed wound healing $\bullet$ Diabetes mellitus $\bullet$ Inflammation - Keratinocytes $\cdot \mathrm{N}$-acetyl-L-cysteine

\begin{abstract}
Background/Aim: Accumulation of advanced glycation end products (AGEs) is a major cause of diabetes mellitus (DM) skin complications. Methylglyoxal (MGO), a reactive dicarbonyl compound, is a crucial intermediate of AGEs generation. N-acetyl-L-cysteine (NAC), an active ingredient of some medicines, can induce endogenous GSH and hydrogen sulfide generation, and set off a condensation reaction with MGO. However, there is rare evidence to show NAC can alleviate DM-induced skin injury through inhibition of AGEs generation or toxicity. The present study aimed to observe the effects of NAC on MGO-induced inflammatory injury and investigate the roles of AGEs and its receptor (RAGE) in NAC's dermal protection in human HaCaT keratinocytes. Methods: The cells were exposed to MGO to simulate a high MGO status in diabetic blood or tissues. The content of AGEs in serum or cell medium was measured with ELISA. The protective effects of NAC against MGO-induce injury were evaluated by administration before MGO one hour, in virtue of cell viability, mitochondrial membrane potential, inflammation reaction, nuclear factor (NF)-kB activation, matrix metalloproteinase (MMP)-9 expression, as well as cellular behavioral function. Results: We found the AGEs levels of patients with DM were elevated comparing with healthy volunteers. The in vitro AGEs generation was also able to be enhanced by the exposure of $\mathrm{HaCaT}$ cells to MGO, which reduced dose-dependently cellular viability, damaged mitochondrial function, triggered secretion of interleukin (IL)- 6 and IL-8, activated NF-KB and upregulated MMP-9 expression. Furthermore, the exposure caused cellular adhesion and migration dysfunction, as well as collagen type I inhibition. Importantly, before the exposure to MGO, the preconditioning with NAC significantly attenuated MGO-induced AGEs generation, improved cellular viability and mitochondrial function, partially reversed the overexpression of proinflammatory factors and MMP-9, as well as the activation of NF-KB. Lastly, NAC blocked MGO-induced RAGE upregulation, and inhibition of RAGE with its neutralizing antibody significantly alleviated C.-t. Yang and F.-h. Meng contributed equally to this study.
\end{abstract}


Yang et al.: Dermal Protection of NAC via Inhibition of AGEs/RAGE

MGO-induced NF-kB activation, MMP-9 upregulation and inflammatory injury in $\mathrm{HaCaT}$ cells. Conclusion: The present work indicates the administration of NAC can prevent MGO-induced dermal inflammatory injury through inhibition of AGEs/RAGE signal, which may provide a basal support for the treatment of diabetic skin complications with NAC-containing medicines in the future.

(C) 2017 The Author(s)

Published by S. Karger AG, Basel

\section{Introduction}

Diabetes mellitus (DM) represents a significant and escalating worldwide health burden, and its number has risen from 108 million in 1980 to 422 million in 2014. In other words, its global prevalence among adults over 18 years of age has risen from $4.7 \%$ in 1980 to $8.5 \%$ in 2014 , which is especially prominent in the developing countries including China. Skin complications, such as delayed wound healing and diabetic foot, account for the major part of various diabetic complications [1]. Luckily, these complications are sometimes the first sign of DM patients. Therefore, it is very necessary to reveal the source of DM condition for the prevention of diabetic skin complications.

Accumulating evidences suggest that advanced glycation end products (AGEs) are one of the most important reasons for diabetic complications [2-4]. In addition, the elevated AGEs levels in blood and tissues are the main reasons for "hyperglycemic memory", because AGEsmediated oxidative stress, inflammatory injury and defective angiogenesis can last a relatively long time although hyperglycemia status has been normalized $[4,5]$. Therefore, it will be reasonable to prevent various diabetic complications by inhibiting AGEs generation. As we known, the endogenous AGEs derive from the abnormal glucose metabolism. Especially, in polyol pathway, the highly reactive dicarbonyl compounds, including methylglyoxal (MGO), glyoxal (GO) and 3-deoxyglucosone, play very important roles [4, 6]. By means of some special medicines or compounds to lower the levels of intermediates, we may thus inhibit AGEs generation and consequent injury under diabetic condition. Our previous studies have shown that MGO-mediated injury in skin keratinocytes and Schwann neural cells was significantly reversed by the donation of hydrogen sulfide $\left(\mathrm{H}_{2} \mathrm{~S}\right)$ or its endogenous induction with some clinical medicines [7, 8].

$\mathrm{N}$-acetyl-L-cysteine (NAC) is a main active ingredient of the clinical common medicines Fluimucil used as an expectorant [9] and Acetylcysteine Injection also known as Ah Si Xintai in Chinese used in the early phase of liver failure through increasing reduced glutathione (GSH) generation and inhibiting oxidative injury of mitochondria [10]. In chemistry, NAC is derived from L-cysteine, whose amino-group is acetylated. It was reported that L-cysteine, as well as its metabolite $\mathrm{H}_{2} \mathrm{~S}$, is decreased in African Americans with Type 2 DM [11, 12]. Another report suggested that NAC can react with MGO to produce N- $\alpha$-acetyl-S-(1-hydroxy-2-oxoprop-1-yl)cysteine [13]. Therefore, the administration of those medicines containing NAC may benefit diabetic patients by reducing the content of highly reactive intermediates and consequently inhibiting AGEs generation and diabetic injury. Of course, NAC can also exert antioxidative effects. For instance, a study has shown that NAC can improve wound healing in alloxan-induced diabetic mice via clearance of free oxygen radicals [14]. In contrast, we notice in some other papers that the levels of plasma total cysteine correlates positively with the obesity or fat mass of patients [15-17]. A recent study suggested that the increased concentration of L-cysteine can inhibit insulin secretion from pancreatic $\beta$-cells through binding and inactivation of pyruvate kinase muscle isoform 2 [18]. With these conflicting reports, we intend to explore the effects of NAC on AGEs generation and diabetic wound healing.

In AGEs-induced diabetic injury, its receptor, receptor for advanced glycation end products (RAGE), usually plays a crucial role in activation of nuclear factor (NF)- $\mathrm{BB}$, secretion of proinflammatory cytokines, such as interleukin (IL)-6 and IL-8, as well as overexpression of matrix metalloproteinase (MMP)-9 [19-22]. Moderate oxidation and inflammation 
Yang et al.: Dermal Protection of NAC via Inhibition of AGEs/RAGE

are conducive to wound healing through induction of proliferation and migration of skin keratinocytes and fibroblasts. However, their excessive or persistent status can hinder wound healing by activation of MMPs or induction of neutrophil NETosis and damage extracellular matrix (ECM) $[23,24]$. Therefore, we want to further investigate whether the effects of NAC on MGO-induced inflammatory injury are associated with the inhibition of AGEs/RAGE signal.

\section{Materials and Methods}

\section{Clinical data}

Peripheral blood was collected from 8 diabetic outpatients (Diabetic group) in the $4^{\text {th }}$ Affiliated Hospital of Guangzhou Medical University. Meanwhile, another 8 healthy volunteers (Control group) were included. The average age of both groups was $(59.3 \pm 8.2$ vs $56.5 \pm 7.4$, respectively, $P=0.49)$ years. In each group, there was the same number of male and female persons. All the experimental operation was approved by Ethics Committee of Guangzhou Medical University and agreed by the subjects.

\section{Materials}

MGO and NAC were bought from Sigma-Aldrich Co. (St. Louis, MO, US). Cell counting kit (CCK)-8 and rhodamine123 (Rh123) were supplied by Dojindo Laboratory (Kyushu, Japan). Commercial enzyme-linked immunosorbent assay (ELISA) kits were provided by Boster BioTech (Wuhan, China) for measurement of IL-6 and IL-8. ELISA kit for AGEs was bought from Cloud-Clone Corp. (Wuhan, China). ELISA kits for ECM, including collagen type I (COL-1) and laminin 5 (LN-5), were provided by Cusabio Biotech Co. Ltd. (Wuhan, China). Primary antibody against RAGE (R\&D Systems Inc. Minneapolis, US), MMP-9 (Abcam Plc. Cambridge, MA, US) or GAPDH (KangChen BioTech, Shanghai, China) was used for Western blot assay. NF- $\mathrm{B}$ p65 subunit was bought from Cell Signaling Technology Inc. (Shanghai, China). Dulbecco's modified Eagle's medium (DMEM) was bought from Gibico BRL (Shanghai, China). Gemcell TM fetal bovine serum (FBS) was supplied by Gemini Company (Woodland, US).

Cell culture

HaCaT cells are derived from spontaneous transformation of human adult keratinocytes, which were provided by Kunming Institute of Zoology in Chinese Academy of Sciences (Kunming, China). The cells were maintained in DMEM supplemented with $10 \% \mathrm{FBS}$ at $37^{\circ} \mathrm{C}$ under an atmosphere of $5 \% \mathrm{CO}_{2}$ and $95 \%$ air.

Measurement of AGEs levels

Serum content of AGEs in the peripheral blood and the cell culture supernatant was detected with ELISA. The peripheral blood was collected from the diabetic patients and healthy volunteers and then the serum was isolated. HaCaT cells were exposed to $400 \mu \mathrm{M} \mathrm{MGO}$ for $48 \mathrm{~h}$ in the absence or presence of 200 $\mu \mathrm{M}$ NAC preconditioning for one hour and then the cell culture supernatant was collected. Then ELISA was performed according to its kit instruction. The absorbance $(A)$ was measured at $450 \mathrm{~nm}$ with a microplate reader (Molecular Devices, US). The AGEs content was calculated in the light of the standard curve.

\section{Determination of cell viability}

HaCaT cells were inoculated in 96-well plates with 4 wells in each group and grown to approximately $70 \%$ confluence. And then the cells were treated with various concentrations of MGO for $48 \mathrm{~h}$ in the absence or presence of pretreatment with NAC or neutralizing antibody against RAGE (NAB-R). After the treatments, cell viability assay was performed. Briefly, $100 \mu \mathrm{L}$ of CCK-8 solution at a 1:10 dilution with FBS-free DMEM was added to each well followed by a 3 -h incubation at $37^{\circ} \mathrm{C}$. The $A$ value was measured at $450 \mathrm{~nm}$ with a microplate reader (Molecular Devices, US). The mean $A$ value of 4 wells in each group was used to calculate percentage of cell viability as follows: Cell viability (\%) $=\left(A_{\text {treatment group }}-A_{\text {Blank }}\right) /\left(A_{\text {control group }}-A_{\text {Blank }}\right) \times 100$. The experiments were performed for 4 times with similar outcomes.

\section{Measurement of mitochondrial function}

Mitochondrial function was assessed on the basis of mitochondrial membrane potential ( $\Delta \Psi \mathrm{m})$, which was tested by Rh123 staining followed by photofluorography. Rh123 is a cell-permeable cationic fluorescent dye, entering mitochondria based on the highly negative $\Delta \Psi \mathrm{m}$. After the indicated treatments, $10 \mathrm{mg} / \mathrm{L} \mathrm{of}$ Rh123 solution prepared with FBS-free medium was added and incubated with HaCaT cells in the dark 


\section{Cellular Physiology Cell Physiol Biochem 2017;41:742-754

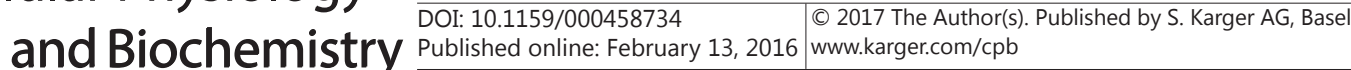

Yang et al.: Dermal Protection of NAC via Inhibition of AGEs/RAGE

at $37^{\circ} \mathrm{C}$ for $30 \mathrm{~min}$. The fluorescent signal was visualized under an fluorescent microscope (Advanced Microscopy Group, US). The mean fluorescence intensity (MFI) of Rh123 was analyzed with Image J software.

\section{ELISA for secretion of proinflammatory factors and ECM}

HaCaT cells were treated with $400 \mu \mathrm{M}$ MGO for $48 \mathrm{~h}$ in the absence or presence of preconditioning with NAC or NAB-R. At the end of the treatments, ELISA was performed to test the content of proinflammatory factors (IL-6 and IL-8), as well as ECM (COL-1 and LN-5) in cell medium according to the manufacturers' instructions. The mean $A$ value was detected with a microplate reader (Molecular Devices, US). Their amount in cell medium was normalized with cell viability.

\section{Western blot assay for RAGE and MMP-9 expression}

After the indicated treatments, HaCaT cells were harvested and split at $4^{\circ} \mathrm{C}$. Thirty microgram of total protein sample was loaded on SDS-polyacrylamide gel electrophoresis (PAGE) (12\% separating gel and $5 \%$ stacking gel). After electrophoresis, the proteins were transferred to polyvinylidene fluoride (PVDF) membranes. The membranes were blocked with 5\% fat-free milk in Tris base buffered saline containing $0.1 \%$ Tween-20 (TBS-T) for one hour at room temperature, and then incubated with the primary antibody against MMP-9 (1:2000), RAGE (1:1000) or GAPDH (1:6000) with gentle agitation overnight at $4^{\circ} \mathrm{C}$. After 3 washes, the horseradish peroxidase-conjugated secondary antibodies (1:4000) were then applied at room temperature for 1.5 hours. In addition, because the difference in molecular weight of RAGE and GAPDH proteins is very small, we used a commercial stripping buffer to remove RAGE primary antibody in the PVDF membrane, followed by blocking and incubation of GAPDH antibody. The signals were visualized through an enhanced chemiluminescence detection system. The intensity of bands was quantified using Image J software.

\section{Confocal microscopy for $N F-\kappa B$ p 65 subunit nuclear translocation}

HaCaT cells were inoculated onto confocal dishes, and cultured overnight. When grown to about $60 \%$ confluence, the cells were treated with $400 \mu \mathrm{M}$ MGO for $24 \mathrm{~h}$ in the absence or presence of preconditioning with NAC or NAB-R. Then, they were rinsed twice with PBS and fixed with methanol for 20 min at room temperature. After permeabilization of the cells with $0.3 \%$ Triton X-100, the dishes were blocked in 5\% BSA for one hour at room temperature, followed by an incubation with p65 subunit primary antibody (1:200) with gentle agitation overnight at $4^{\circ} \mathrm{C}$. The secondary antibody of FITC-conjugated IgG (1:50) was incubated with the cells for one hour. After 3 washes with PBS, the samples were mounted with DAPI and then were visualized under a laser-scanning confocal microscopy (Carl Zeiss AG, German). Double staining (Merge) for FITC and DAPI was performed to display the nuclear translocation of NF- $\kappa$ B p 65 subunit.

\section{Cellular adhesion assay}

HaCaT cells were treated with $400 \mu \mathrm{M}$ MGO for $48 \mathrm{~h}$ in the absence or presence of preconditioning with $200 \mu \mathrm{M}$ NAC for 1 hour. At the end of the treatments, cellular adhesion assay was performed referring to our previous paper [7]. Briefly, the cells were digested with $0.25 \%$ trypsin and centrifuged at $1500 \mathrm{rpm}$ for $5 \mathrm{~min}$. The harvested cells were inoculated on 96-well plates, with 8 wells in each group. The CCK-8 solution at a 1:10 dilution was added to 4 of the wells in each group to measure the total cell number. The cells in another 4 wells were cultured for a further $12 \mathrm{~h}$. The cell medium was removed and the plate wells were washed with PBS to remove the unattached cells. The same volume of CCK-8 solution was added as above to measure the adhesive cell number. The adhesion rate $=$ the adhesive cells $/$ the total cells $\times 100 \%$.

Cellular migration assay

Cellular migration was observed with an in vitro scratch healing assay performed as described previously with certain modifications [7]. HaCaT cells were exposed to $400 \mu \mathrm{M}$ MGO for $48 \mathrm{~h}$ in the absence or presence of pretreatment with $200 \mu \mathrm{M}$ NAC for $1 \mathrm{~h}$. After the treatments, the cells were inoculated on 6 -well plates and cultured up to about 75\% confluence. A narrow wound-like gap in the cell monolayer was created with a pipette tip, the shedding cells were washed off with PBS and then the images were captured. The cells were further suffered from the above treatments for a further $12 \mathrm{~h}$ and the cell images were captured again. 
Statistical analysis

All the data were presented as the means \pm standard deviation (SD). The significance of inter-group was evaluated by one-way analysis of variance (ANOVA) followed by Student-Newman-Keuls test with SPSS software. $P<0.05$ was considered to be statistically significant.

\section{Results}

Content of AGEs in diabetic patients was elevated

The elevated AGEs content is a main cause of diabetic complications. Therefore, we firstly performed experiment to examine the serum AGEs levels with ELISA. As shown in Fig. 1 , the content of serum AGEs was significantly higher in the diabetic patients than those in the control healthy volunteers.

\section{NAC inhibited MGO-induced AGEs generation}

Since MGO is an important reactive intermediate of AGEs generation, its elimination may hinder AGEs produce in biological systems. Fig. 2a showed a possible chemical reaction between MGO and NAC, by which we surmised NAC should reduce MGO content, inhibit AGEs generation and consequent cellular injury. We then performed experiment to demonstrate this hypothesis. As shown in Fig. 2b, after human skin HaCaT cells were exposed to MGO, the AGEs produce was significantly increased in cell medium. However, the MGO-induced AGEs generation was markedly inhibited by the pretreatment with NAC.

\section{NAC attenuated MGO-induced cellular injury}

Now that NAC was able to inhibit the MGO-stimulated AGEs generation, we expected that it could exert a protective action against MGO-induced injury. With this idea in mind, we tested cell viability with CCK-8 assay. The result of Fig. 3a displayed that the exposure of HaCaT cells to MGO induced a dose-dependent inhibition of viability. In addition, the result also indicated that the median lethal concentration of MGO was about $400 \mu \mathrm{M}$ in HaCaT cells. Importantly, before the exposure to MGO, the cells were pretreated with NAC at various concentrations for one hour. As expected, we found that the pretreatment

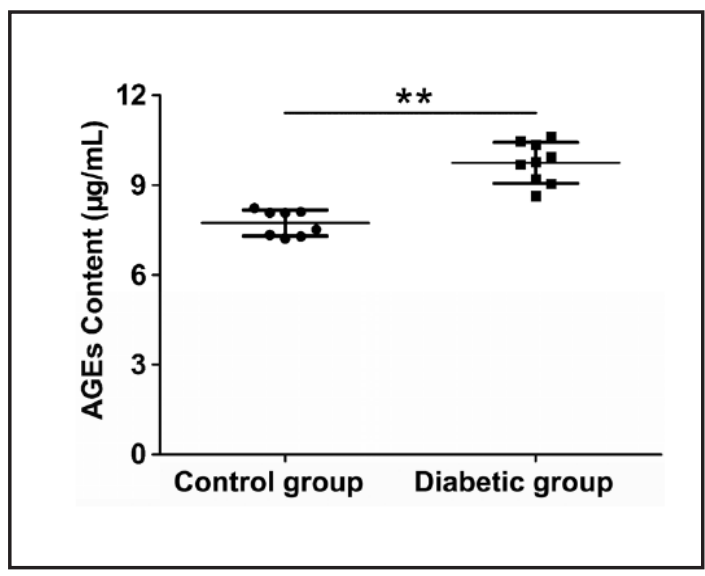

Fig. 1. Analysis of serum AGEs content in diabetic patients and healthy volunteers. The peripheral blood was collected and then the serum was isolated. The serum AGEs content was detected with ELISA. Data were shown as the mean \pm SD. $n=8,{ }^{* *} P<0.01$ versus Control group.

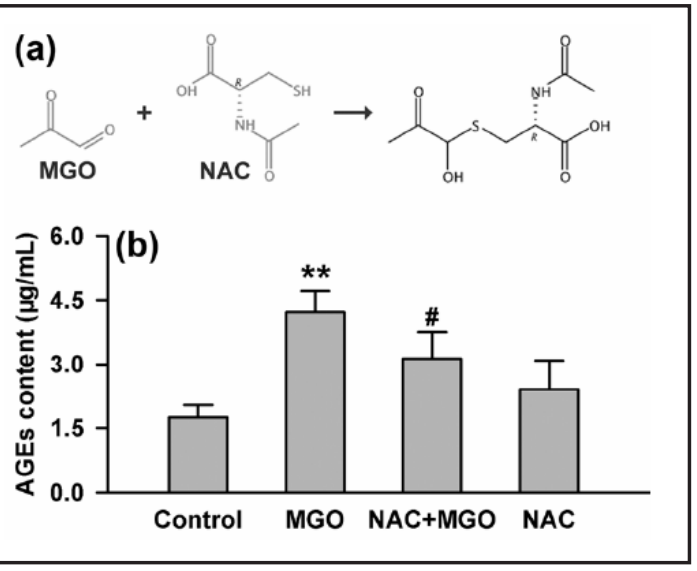

Fig. 2. Effects of NAC on MGO-induced AGEs generation. (a) A possible chemical reaction between MGO and NAC. (b) HaCaT cells were exposed to $400 \mu \mathrm{M}$ MGO for $48 \mathrm{~h}$ in the presence or absence of the pretreatment with $200 \mu \mathrm{M}$ NAC for $1 \mathrm{~h}$. After the treatments, the cell medium was collected and the content of AGEs was measured with ELISA. Data were shown as the mean \pm SD. ${ }^{* *} P<0.01$ versus Control group, ${ }^{\#} P<0.05$ versus MGO alone group.

\section{KARGER}




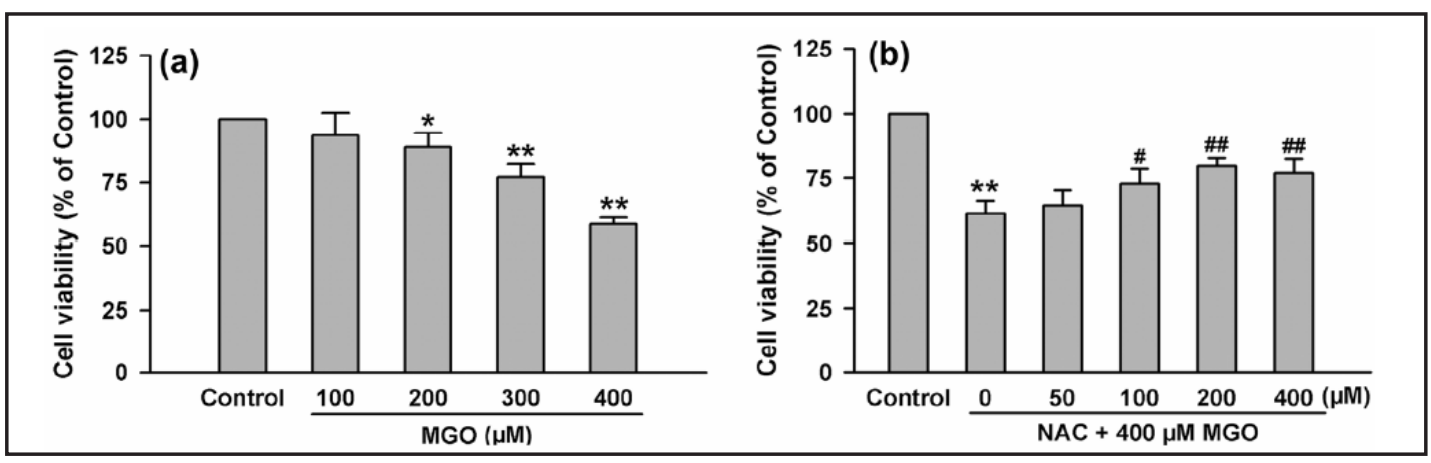

Fig. 3. Effects of NAC on MGO-induced cell injury in HaCaT cells. (a) The cells were exposed to the increasing concentrations of MGO from $100 \mu \mathrm{M}$ to $400 \mu \mathrm{M}$ for $48 \mathrm{~h}$. (b) Before the exposure to $400 \mu \mathrm{M}$ MGO, the cells were incubated with various concentrations of NAC for $1 \mathrm{~h}$. After the treatments, the cell viability was measured with CCK-8 assay. Data were shown as the mean \pm SD. $n=4 .{ }^{*} P<0.05,{ }^{* *} P<0.01$ versus Control group, ${ }^{\#} P<0.05$, ${ }^{\# \# ~} P<0.01$ versus MGO alone group.

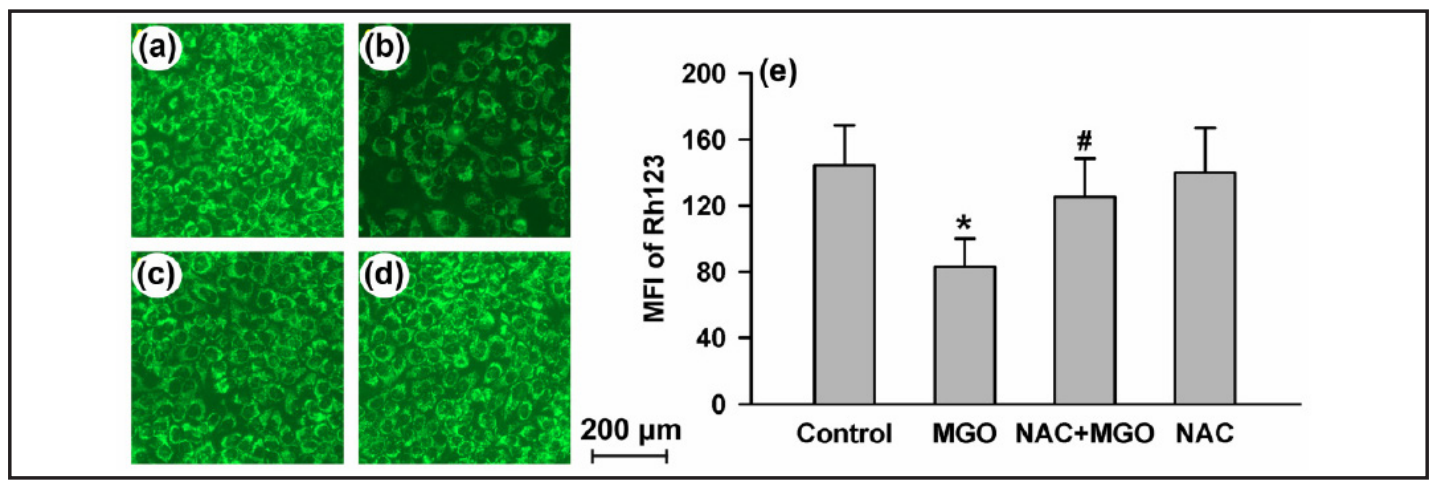

Fig. 4. Effects of NAC on MGO-induced mitochondrial injury in HaCaT cells. The random fluorescence micrographs from the indicated cells: (a) control cells, (b) cells treated with $400 \mu \mathrm{M}$ MGO for $48 \mathrm{~h}$, (c) cells treated with MGO in the presence of preconditioning with $200 \mu \mathrm{M} \mathrm{NAC}$ for $1 \mathrm{~h}$ and (d) control cells treated with NAC for $1 \mathrm{~h}$ followed by a $48 \mathrm{~h}$ culture. (e) Quantitative analysis of the mean fluorescence intensity (MFI) of Rh123 using Image J software. Data were shown as the mean \pm SD. $n=4 .{ }^{*} P<0.05$ versus control group, ${ }^{\#} P<0.05$ versus MGO alone group.

with NAC significantly blunted MGO-induced injury in HaCaT cell (Fig. 3b), indicating the cytoprotective effects of NAC against MGO-related cellular injury.

\section{NAC impeded MGO-triggered mitochondrial injury in HaCaT cells}

Mitochondria are a very important organelle and prone to be damaged by diabetes. The depolarization of $\Delta \Psi \mathrm{m}$ is an early evidence of the mitochondrial dysfunction. The levels of $\Delta \Psi \mathrm{m}$ can be determined by a fluorescent dye Rh123 entry. The results showed that the exposure of HaCaT cells to $400 \mu \mathrm{M}$ MGO obviously suppressed the $\Delta \Psi \mathrm{m}$ levels, characterized by a weaker Rh123 fluorescence (Fig. 4b) than that in the control cells (Fig. 4a). Prior to the exposure to MGO, the cells were pretreated with $200 \mu \mathrm{M}$ NAC for one hour. The results further showed the pretreatment with NAC distinctly impeded MGO-triggered $\triangle \Psi$ m loss (Fig. 4c), without altering the $\Delta \Psi \mathrm{m}$ levels in the quiescent cells (Fig. 4d). The results suggest that the NAC-mediated mitochondrial protection may contribute to its cytoprotection.

\section{NAC suppressed inflammation response in HaCaT cells}

Diabetes is a chronic and excessive inflammation response, delaying wound healing. We examined some of the proinflammatory factors, IL-6 and IL-8, with ELISA. The results of Fig. 5a presented that the exposure of HaCaT cells to $400 \mu \mathrm{M}$ MGO for $48 \mathrm{~h}$ significantly

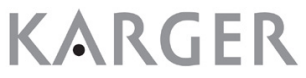




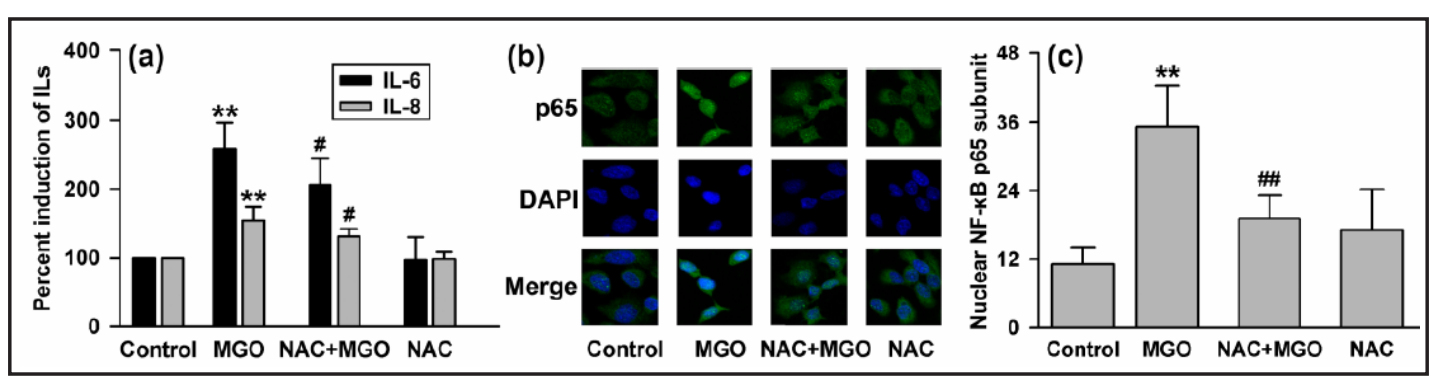

Fig. 5. Effects of NAC on inflammation response in MGO-stimulated HaCaT cells. The cells were preconditioned with $200 \mu \mathrm{M} \mathrm{NAC}$ for $1 \mathrm{~h}$ and then exposed to $400 \mu \mathrm{M}$ MGO for $48 \mathrm{~h}$. After the treatments, (a) the content of IL- 6 and IL-8 in cell medium was detected with ELISA, (b) NF- $\kappa B$ p65 subunit content in the nuclei was observed with immunocytochemical technique under a confocal microscopy and (c) Quantitative analysis of the MFI of p65 subunit using Image J software. Data were shown as the mean $\pm \mathrm{SD} . \mathrm{n}=4 .{ }^{* *} P<0.01$ versus control group, ${ }^{\#} P<0.05$, ${ }^{\#} P<0.01$ versus MGO alone group.

Fig. 6. Effects of NAC on MMP-9 expression in MGO-treated HaCaT cells. The cells were exposed to $400 \mu \mathrm{M}$ MGO for $48 \mathrm{~h}$ in the presence or absence of the pretreatment with $200 \mu \mathrm{M}$ NAC for $1 \mathrm{~h}$. After the treatments, the cells were collected and split, and the total proteins were used to detect MMP-9 with Western blot analysis (a). Panel b was the result of densitometric analysis from Panel a. Data were shown as the mean $\pm \mathrm{SD} .{ }^{* *} P<0.01$ versus Control group, ${ }^{\# \#} P<0.01$ versus $\mathrm{MGO}$ alone group.

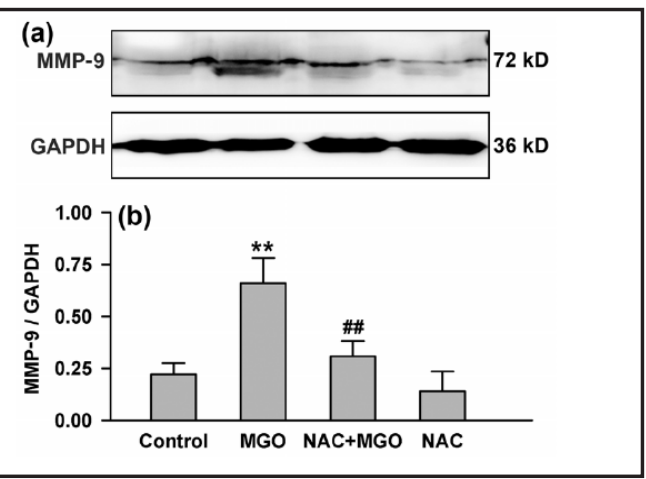

enhanced their secretion. However, the secretion induced by MGO was suppressed by the pretreatment with $200 \mu \mathrm{M}$ NAC for one hour, which alone didn't change inflammation levels (Fig. 5a). In addition, NF- $\kappa \mathrm{B}$ is an important transcription factor regulating expression of many proinflammatory factors. Nuclear translocation of p65 subunit is a critical step of NF$\kappa \mathrm{B}$ activation. We used a confocal microscopy to observe nuclear $\mathrm{p} 65$ protein and found that the exposure to MGO clearly enhanced the p65 levels in the nuclei, which was also decreased by the pretreatment with NAC (Fig. $5 b$ and c). The results indicate that NAC may extenuate inflammatory response through inhibition of NF- $\mathrm{BB}$ activation under diabetic condition.

\section{NAC impeded MGO-induced MMP-9 upregulation in HaCaT cells}

Overexpression of MMP-9 is a significant reason for impaired diabetic wound healing, and can be regulated by NF- $\mathrm{BB}$. The result of Fig. 6 displayed that the treatment with $400 \mu \mathrm{M}$ MGO for $48 \mathrm{~h}$ distinctively induced MMP-9 overexpression. However, this effect was impeded by the pretreatment with $200 \mu \mathrm{M}$ NAC for 1 hour, which alone didn't alter MMP-9 expression. The result indicates that NAC may improve diabetic wound healing.

\section{NAC restored adhesion and migration in MGO-treated HaCaT cells}

Proper behavioral function of keratinocytes, including adhesion and migration, is very critical for skin wound healing. By testing cell adhesion function, we found that the treatment with $400 \mu \mathrm{M}$ MGO for $48 \mathrm{~h}$ significantly reduced the number of adhesive cells (Fig. 7a) and adhesion rate (Fig. $7 \mathrm{~b}$ ) comparing with control group. However, the inhibition of adhesion induced by MGO was markedly prevented by the pretreatment with $200 \mu \mathrm{M}$ NAC for one hour (Fig. 7a and b). Additionally, we observed keratinocyte migration function with an in vitro scratch healing assay. The result of Fig. $7 \mathrm{c}$ and d showed that the exposure of HaCaT cells to $400 \mu \mathrm{M}$ MGO for $48 \mathrm{~h}$ markedly suppressed cell migration. However, the impaired cell migration induced by MGO was alleviated by the pretreatment with NAC, which alone didn't significantly affect the cellular migration in HaCaT cells. The results suggest that NAC 

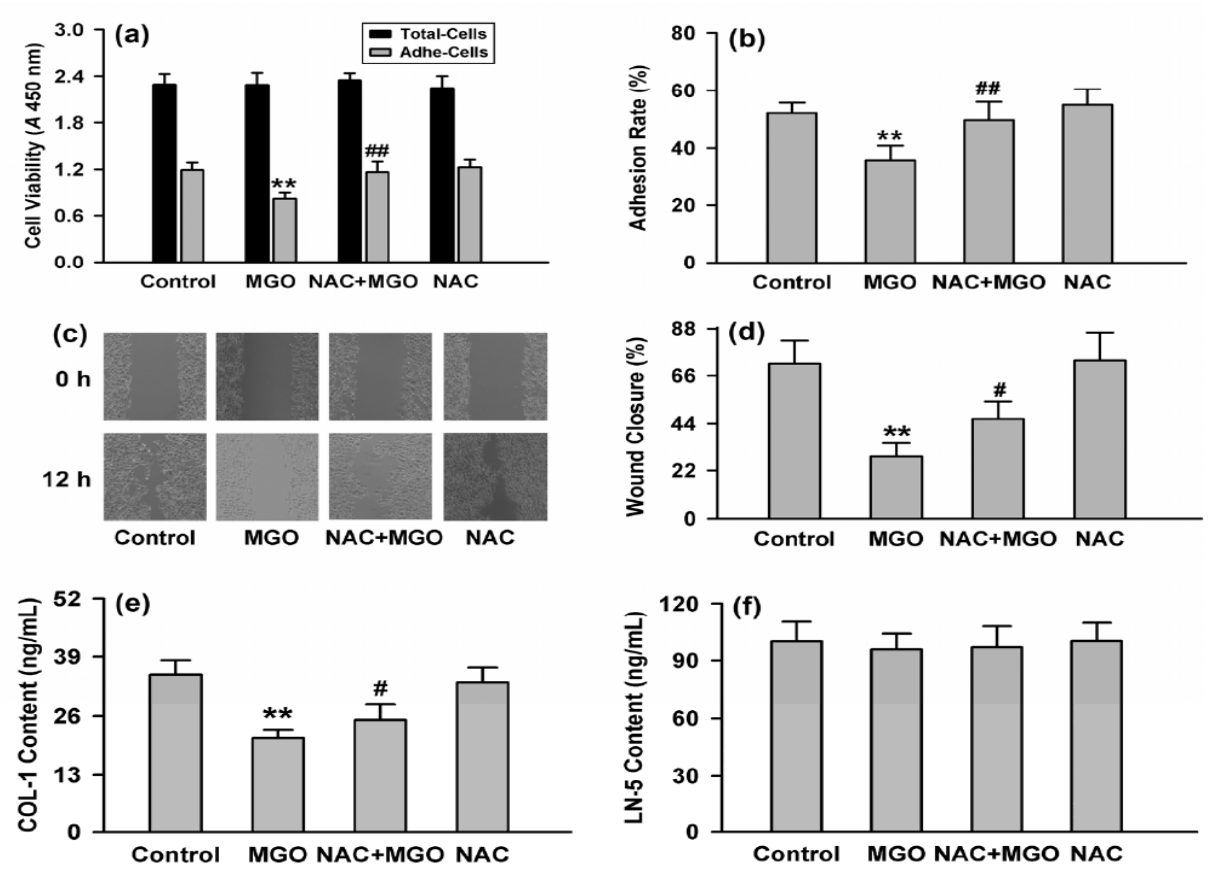

Fig. 7. Effects of NAC on cellular adhesion and migration in MGO-treated HaCaT cells. The cells were cultured with $400 \mu \mathrm{M}$ MGO for $48 \mathrm{~h}$ in the absence or presence of preconditioning with $200 \mu \mathrm{M} \mathrm{NAC}$ for $1 \mathrm{~h}$. After the treatments, the cells were digested with $0.25 \%$ trypsin and collected. (a) The harvested cells were inoculated on two 96-well plates at the same density and cultured $12 \mathrm{~h}$. The numbers of total cells and adhesive cells was measured with CCK-8 assay. (b) Adhesion rate (\%) was calculated with a ratio of adhesion cells to total cells. (c) The harvested cells were inoculated on 6-well plates and cultured up to $75 \%$ confluence. Scratch healing assay was performed to observe cell migration in vitro. The images were captured at the time of scratch $(0 \mathrm{~h})$ and $12 \mathrm{~h}$ after the scratch. (d) Wound closure (\%) was calculated with a ratio of the area of $0 \mathrm{~h}$ to the area of $12 \mathrm{~h}$, measured with Image $\mathrm{J}$ software. The cell medium was collected and the ECM contents of COL-1 (e) and LN-5 (f) were tested with ELISA. Data were shown as the mean \pm SD. ${ }^{* *} P<0.01$ versus control group; ${ }^{\#}<0.05$, ${ }^{\#} P<0.01$ versus MGO alone group.

is benefical for in vitro skin wound healing. We further investigated the related mechanisms and found the effects of NAC were associated with the restoration of COL-1 (Fig. 7e) and might not be associated with LN-5 expression (Fig. 7f).

Dermal protection of NAC was associated with inhibition of AGEs-mediated RAGE expression

As shown in Fig. 8a, the exposure of HaCaT cells to $400 \mu \mathrm{M}$ MGO for 48 h significantly upregulated the expression of AGEs receptor, RAGE. However, the result further showed the induction of RAGE was attenuated by the pretreatment with $200 \mu \mathrm{M} \mathrm{NAC}$ for $1 \mathrm{~h}$. Furthermore, we found that the blockage of RAGE with its neutralizing antibody (NAB-R) prevented MGOinduced cellular injury (Fig. 8c), secretion of IL-6 and IL-8 (Fig. 8d), overexpression of MMP-9 (Fig. 8e and f), as well as NF- $\mathrm{BB}$ activation (Fig. 8g and $\mathrm{h}$ ). These results suggest that MGO-triggered RAGE upregulation is involved in its proinflammation and cellular injury, and inhibition of RAGE may be an important mechanism underlying NAC's anti-inflammatory injury.

\section{Discussion}

In the current study, we showed that the diabetic patients had relatively high levels of serum AGEs comparing with the healthy volunteers. The in vitro administration of NAC 
(a)
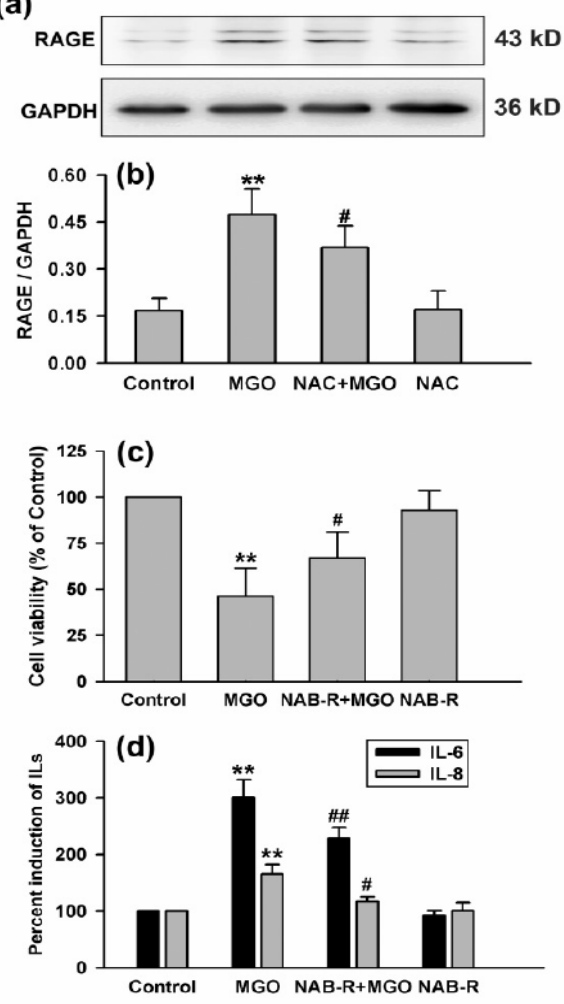

(e)
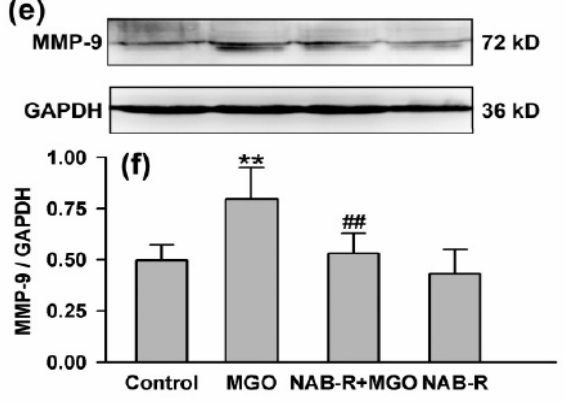

(g)
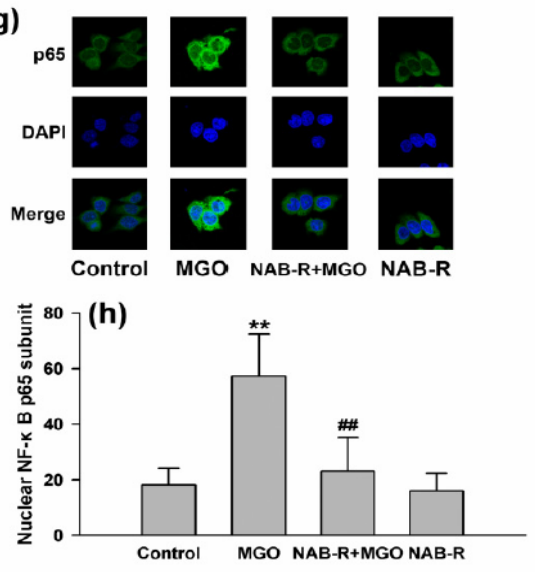

Fig. 8. Roles of RAGE in prevention of NAC against MGO-induced inflammatory injury. (a) HaCaT cells were exposed to $400 \mu \mathrm{M}$ MGO for $48 \mathrm{~h}$ in the presence or absence of pretreatment with $200 \mu \mathrm{M}$ NAC for $1 \mathrm{~h}$. After the treatments, the cells were collected and split, and the total proteins were used for Western blot assay to test RAGE expression. Panel $\mathrm{b}$ was the result of densitometric analysis from Panel a. HaCaT cells were exposed to $400 \mu \mathrm{M}$ MGO in the presence or absence of pretreatment with $0.01 \mathrm{mg} / \mathrm{mL}$ neutralizing antibody against RAGE (NAB-R) for $6 \mathrm{~h}$. At the end of treatments, the cell viability was measured with CCK-8 assay (c), the content of IL-6 and IL-8 in cell medium was detected with ELISA (d), the expression of MMP-9 was measured with Western blot assay (e and f) and the content of NF- $\mathrm{kB}$ p65 subunit in nuclei was observed with immunocytochemical technique under a confocal microscopy ( $g$ and $h$ ). Data were shown as the mean \pm SD. ${ }^{* *} P<0.01$ versus Control group, ${ }^{\#} P<0.05$, ${ }^{\#} P<0.01$ versus MGO alone group.

inhibited MGO-induced AGEs generation in skin keratinocytes. Importantly, the treatment with NAC significantly reduced MGO-triggered inflammatory injury, mitochondrial dysfunction and impairment of cellular behavioral action. Further result displayed that the blockage of NAC on MGO-induced inflammatory injury was mediated by RAGE. Our work indicates that the administration of NAC can attenuate DM related dermal injury through inhibition of AGEs/RAGE signal.

AGEs are accepted as one of the most important causes of various diabetic complications, including diabetic foot and delayed wound healing [25]. Through measurement of the serum AGEs, we found that the AGEs levels were significantly higher in the diabetic patients than those in the healthy volunteers, which was consistent with the previous report [26]. It is logical to dissect the process of AGEs generation for discovering some therapeutic targets of diabetic skin complications. In diabetes, reactive dicarbonyl substances, especially MGO, were closely associated with AGEs generation [27]. Therefore, it may be an ideal therapeutic strategy to inhibit pharmacologically the reaction between the reactive dicarbonyl substances and biomacromolecules with some commonly used medicines. One of the most important examples is aminoguanidine, which is a raw material of some medicines. 
A study has shown that aminoguanidine can block DM-induced cross-link of arterial wall connective tissue protein and AGEs formation in vivo [28]. NAC used in the present study is a raw material and main active ingredient of clinical medicines Fluimucil and Acetylcysteine Injection. According to its special chemical structure, NAC will hinder MGO-medicated crosslink and AGEs generation. A previous study has also indicated that NAC can react with MGO to produce $\mathrm{N}$ - $\alpha$-acetyl-S-(1-hydroxy-2-oxo-prop-1-yl)cysteine [13]. Our result showed the in vitro administration of NAC suppressed MGO-induced AGEs generation in HaCaT cells.

Inhibition of AGEs generation, we surmise, may blunt MGO-induced a variety of injury. With our previous experiment model [7], we found that the treatment of HaCaT cells with MGO dose-dependently reduced cell viability. However, MGO-induced cell injury was significantly attenuated by the pretreatment with NAC. Of note, the concentration $(200 \mu \mathrm{M})$ of NAC used in this study is far below the concentration $(2000 \mu \mathrm{M})$ used as an antioxidant [29]. In addition, in this study we chose a pretreatment method, in other words, the cells were pretreated with NAC before the exposure to MGO. At the end of NAC pretreatment, the cell medium was discarded and the cells were washed with PBS. Therefore, we believed that NAC-mediated dermal protection was not just a simple extracellular chemical reaction. The mechanisms may underlie an intracellular condensation reaction between NAC and MGO, as well as regulation of signaling pathway. The former was demonstrated by our data and Wild's experiment [13]. A recent study showed that aminoguanidine cream attenuated oxidation and inflammation levels in skin tissue of diabetic rats through inhibition of AGEs generation [3]. However, we think that the protective mechanisms of NAC may be more complex than those of aminoguanidine, because its index compound (L-cysteine) is also involved in some endogenous gaseous messenger generation, such as $\mathrm{H}_{2} \mathrm{~S}$ and sulfur dioxide $\left(\mathrm{SO}_{2}\right)$, as well as antioxidant GSH in the body [10, 30, 31].

Mitochondria are a vital organelle and easily damaged by various noxious stimuli. Under diabetic condition, mitochondria are usually suffered from oxidative injury. Therefore, improvement of mitochondrial function has become an important therapeutic strategy of diabetic complications [32]. The depolarization of membrane potential happens relatively early when the cells are insulted, and are usually used as an index of cell injury. Through testing the membrane potential, we found that the exposure to MGO markedly decreased $\Delta \Psi \mathrm{m}$ levels, evidenced by a weak green fluorescence in the cells, indicating mitochondrial injury. Ray et al. showed MGO inhibits electron flow through complex I leading to mitochondrial dysfunction $[33,34]$. In addition, some reports revealed that AGEs can also trigger mitochondrial dysfunction $[35,36]$. These studies provide a strong support for our study about MGO-induced mitochondrial dysfunction. However, our following study indicated that this mitochondrial injury induced by MGO was partially reversed by the preadministration of NAC. According to these reports and our findings, we therefore believe that NAC may alleviate diabetic skin injury through improvement of mitochondrial function.

Although inflammation response at the early stage of a wound is beneficial to skin wound healing through stimulating migration and proliferation of fibroblasts and keratinocytes around skin wound, the sustained or excessive inflammation is known to impede wound healing. In this study, we found that the exposure of HaCaT cells to MGO caused remarkable inflammation by increasing secretion of IL- 6 and IL-8. A research even indicated that high levels of glucose also increased IL-8 release from keratinocytes [37]. Both this report and our finding support that the abnormal metabolism of glucose and high levels of MGO can induce inflammation of keratinocytes, which will be harmful for diabetic wound healing. We think that the proinflammatory effects of high glucose or MGO may partially imitate diabetic inflammatory reaction. In addition, the sustained inflammation usually triggers MMPs overexpression, which can degrade extracellular matrix (ECM) and delay diabetic wound healing. In this study, we measured MMP-9 and found that the MGO exposure distinctly elevated its expression. In addition, since the expression of proinflammatory factors and MMP-9 was regulated by NF- $\kappa B$ [22], we investigated the activation of NF- $\kappa$ B by observing its subunit 655 nuclear translocation. The result showed that the exposure to MGO induced obvious p65 nuclear translocation indicating NF- $\mathrm{BB}$ activation. Importantly, we found that

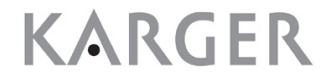




\section{Cellular Physiology Cell Physiol Biochem 2017;41:742-754

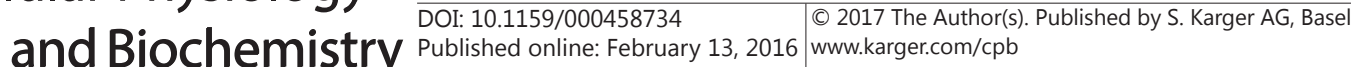

Yang et al.: Dermal Protection of NAC via Inhibition of AGEs/RAGE

MGO-induced secretion of IL-6 and IL-8, as well as overexpression of MMP-9 was attenuated by the pretreatment with NAC. These findings indicated that NAC may be useful for the treatment of the diabetic wound healing through inhibition of intense inflammation.

An appropriate behavioral action of skin keratinocytes, including proliferation, adhesion and migration, is very necessary for wound healing under normal conditions. However, this action becomes weak under the diabetic condition and then delays wound healing. A research has indicated that the keratinocytes of diabetic ulcer edge have high proliferation, but significantly low ability of differentiation and migration comparing with the healthy volunteers [38]. Our previous paper showed the exposure to MGO brought on the impairment of adhesion and migration in skin keratinocytes [7]. In this study, we demonstrated these effects of MGO again, and also found that MGO-triggered behavioral impairment was significantly attenuated by the preadministration of NAC. Furthermore, two recent studies showed that the improvement of migration by dendritic epidermal $\mathrm{T}$ cells markedly facilitated wound healing of DM $[39,40]$. Moreover, ECM is the basis of adhesion and migration during skin wound healing. Through testing COL-1 and LN-5, we found that the exposure to MGO apparently decreased COL-1 expression, but didn't evidently alter LN-5 level. However, the MGO-medicated inhibition of COL-1 was partially reversed by the pretreatment with NAC. We think this may be associated with NAC's decrease in MMP-9, because MMP-9 can degrade ECM. Based on these reports and our above findings, we are convinced that the administration of NAC may be used for the treatment of diabetic wound healing in the future.

Last but not least, because many of the detrimental effects of AGEs are mediated by its receptor RAGE, we explored the roles of RAGE in this study. The results showed that the exposure of HaCaT cells to MGO markedly upregulated RAGE expression, and the effects of MGO were remarkably depressed by the preadministration of NAC. Importantly, the blockage of RAGE's action with its neutralizing antibody significantly improved cell viability. Simultaneously, it attenuated MGO-induced secretion of proinflammatory factors, upregulation of MMP-9, as well as activation of NF- $\mathrm{KB}$ in HaCaT cells. These data indicate that NAC's inhibiting AGEs generation by trapping MGO may contribute to the inhibition of RAGE induction. Since the overexpression of RAGE activated NF- $\kappa B$, and consequently raised the expression of MMP-9 and interleukins, the inhibition of RAGE would relatively attenuate the expression of these inflammatory molecules and exert dermal protection. These findings were supported by the reports from Zhu and Tikellis [22, 41]. We believed that the in vivo administration of NAC may be protective towards diabetic skin injury through inhibition of AGEs generation and RAGE overexpression in the future.

In conclusion, we found that the exposure of skin cells to MGO induced AGEs/RAGE signal upregulation and inflammatory injury, ultimately leading to behavioral dysfunction of HaCaT cells. Importantly, these detrimental effects were significantly mitigated by the preadministration of NAC. The present study may provide a basal support for the treatment of diabetic skin complications with NAC-containing medicines in the future.

\section{Acknowledgments}

This study was supported by Natural Science Foundation of Guangdong Province in China (2015A030313458), Science \& Technology Planning Project of Guangzhou in China (201510010201), Projects of Colleges \& Universities directly under the Guangzhou Education Bureau (1201410467 and 1201620148) and National Funds for developing local colleges and universities (B16056001). In addition, we thank the anonymous reviewers and the editors for their constructive suggestions in order to improve our manuscript.

\section{Disclosure Statement}

The authors have no potential conflicts of interest to declare. 


\section{Cellular Physiology Cell Physiol Biochem 2017;41:742-754

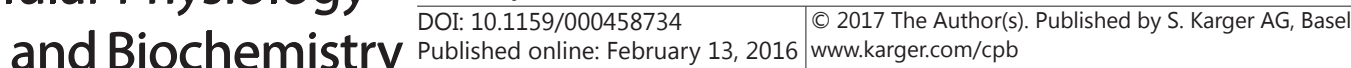

\section{References}

1 Tsourdi E, Barthel A, Rietzsch H, Reichel A, Bornstein SR: Current aspects in the pathophysiology and treatment of chronic wounds in diabetes mellitus. Biomed Res Int 2013;2013:385641.

2 Tian M, Qing C, Niu Y, Dong J, Cao X, Song F, Ji X, Lu S: Effect of aminoguanidine intervention on neutrophils in diabetes inflammatory cells wound healing. Exp Clin Endocrinol Diabetes 2013;121:635-642.

- 3 Tian M, Qing C, Niu Y, Dong J, Cao X, Song F, Ji X, Lu S: Aminoguanidine cream ameliorates skin tissue microenvironment in diabetic rats. Arch Med Sci 2016;12:179-187.

-4 Singh VP, Bali A, Singh N, Jaggi AS: Advanced glycation end products and diabetic complications. Korean J Physiol Pharmacol 2014;18:1-14. Giacco F, Brownlee M: Oxidative stress and diabetic complications. Circ Res 2010;107:1058-1070. Singh R, Barden A, Mori T, Beilin L: Advanced glycation end-products: a review. Diabetologia 2001;44:129146.

7 Yang CT, Zhao Y, Xian M, Li JH, Dong Q, Bai HB, Xu JD, Zhang MF: A novel controllable hydrogen sulfidereleasing molecule protects human skin keratinocytes against methylglyoxal-induced injury and dysfunction. Cell Physiol Biochem 2014;34:1304-1317.

-8 Zhang H, Zhuang XD, Meng FH, Chen L, Dong XB, Liu GH, Li JH, Dong Q, Xu JD, Yang CT: Calcitriol prevents peripheral RSC96 Schwann neural cells from high glucose \& methylglyoxal-induced injury through restoration of CBS/ $\mathrm{H}_{2} \mathrm{~S}$ expression. Neurochem Int 2016;92:49-57.

-9 Sheffner AL: The reduction in vitro in viscosity of mucoprotein solutions by a new mucolytic agent, N-acetyl-L-cysteine. Ann N Y Acad Sci 1963;106:298-310.

10 Saito C, Zwingmann C, Jaeschke H: Novel mechanisms of protection against acetaminophen hepatotoxicity in mice by glutathione and N-acetylcysteine. Hepatology 2010;51:246-254.

11 Jain SK, Kahlon G, Bass P, Levine SN, Warden C: Can L-Cysteine and Vitamin D Rescue Vitamin D and Vitamin D Binding Protein Levels in Blood Plasma of African American Type 2 Diabetic Patients? Antioxid Redox Signal 2015;23:688-693.

12 Jain SK, Bull R, Rains JL, Bass PF, Levine SN, Reddy S, McVie R, Bocchini JA: Low levels of hydrogen sulfide in the blood of diabetes patients and streptozotocin-treated rats causes vascular inflammation? Antioxid Redox Signal 2010;12:1333-1337.

13 Wild R, Ooi L, Srikanth V, Munch G: A quick, convenient and economical method for the reliable determination of methylglyoxal in millimolar concentrations: the N-acetyl-L-cysteine assay. Anal Bioanal Chem 2012;403:2577-2581.

14 Aktunc E, Ozacmak VH, Ozacmak HS, Barut F, Buyukates M, Kandemir O, Demircan N: N-acetyl cysteine promotes angiogenesis and clearance of free oxygen radicals, thus improving wound healing in an alloxaninduced diabetic mouse model of incisional wound. Clin Exp Dermatol 2010;35:902-909.

15 Elshorbagy AK, Nurk E, Gjesdal CG, Tell GS, Ueland PM, Nygard O, Tverdal A, Vollset SE, Refsum H: Homocysteine, cysteine, and body composition in the Hordaland Homocysteine Study: does cysteine link amino acid and lipid metabolism? Am J Clin Nutr 2008;88:738-746.

16 Carter RN, Morton NM: Cysteine and hydrogen sulphide in the regulation of metabolism: insights from genetics and pharmacology. J Pathol 2016;238:321-332.

$\checkmark 17$ El-Khairy L, Vollset SE, Refsum H, Ueland PM: Predictors of change in plasma total cysteine: longitudinal findings from the Hordaland homocysteine study. Clin Chem 2003;49:113-120.

18 Nakatsu D, Horiuchi Y, Kano F, Noguchi Y, Sugawara T, Takamoto I, Kubota N, Kadowaki T, Murata M: L-cysteine reversibly inhibits glucose-induced biphasic insulin secretion and ATP production by inactivating PKM2. Proc Natl Acad Sci USA 2015;112:E1067-1076.

19 Han D, Yamamoto Y, Munesue S, Motoyoshi S, Saito H, Win MT, Watanabe T, Tsuneyama K, Yamamoto H: Induction of receptor for advanced glycation end products by insufficient leptin action triggers pancreatic beta-cell failure in type 2 diabetes. Genes Cells 2013;18:302-314.

20 Neumann A, Schinzel R, Palm D, Riederer P, Munch G: High molecular weight hyaluronic acid inhibits advanced glycation endproduct-induced NF-kappaB activation and cytokine expression. FEBS Lett 1999;453:283-287.

21 Kang R, Tang D, Lotze MT, Zeh HJ, 3rd: RAGE regulates autophagy and apoptosis following oxidative injury. Autophagy 2011;7:442-444. 


\section{Cellular Physiology Cell Physiol Biochem 2017;41:742-754 and Biochemistry DOI: 10.1159/000458734 2016 (O) 2017 The Author(s). Published by S. Karger AG, Basel

22 Zhu P, Ren M, Yang C, Hu YX, Ran JM, Yan L: Involvement of RAGE, MAPK and NF-kappaB pathways in AGEsinduced MMP-9 activation in HaCaT keratinocytes. Exp Dermatol 2012;21:123-129.

23 Gao M, Nguyen TT, Suckow MA, Wolter WR, Gooyit M, Mobashery S, Chang M: Acceleration of diabetic wound healing using a novel protease-anti-protease combination therapy. Proc Natl Acad Sci USA 2015;112:15226-15231.

24 Wong SL, Demers M, Martinod K, Gallant M, Wang Y, Goldfine AB, Kahn CR, Wagner DD: Diabetes primes neutrophils to undergo NETosis, which impairs wound healing. Nat Med 2015;21:815-819.

25 Negre-Salvayre A, Salvayre R, Auge N, Pamplona R, Portero-Otin M: Hyperglycemia and glycation in diabetic complications. Antioxid Redox Signal 2009;11:3071-3109.

26 Nakayama H, Taneda S, Manda N, Aoki S, Komori K, Kuroda Y, Misawa K, Tsushima S, Nakagawa S: Radioimmunoassay for nonenzymatically glycated protein in human serum. Clin Chim Acta 1986;158:293299.

27 Ma H, Li SY, Xu P, Babcock SA, Dolence EK, Brownlee M, Li J, Ren J: Advanced glycation endproduct (AGE) accumulation and AGE receptor (RAGE) up-regulation contribute to the onset of diabetic cardiomyopathy. J Cell Mol Med 2009;13:1751-1764.

28 Brownlee M, Vlassara H, Kooney A, Ulrich P, Cerami A: Aminoguanidine prevents diabetes-induced arterial wall protein cross-linking. Science 1986;232:1629-1632.

29 Yang C, Ling H, Zhang M, Yang Z, Wang X, Zeng F, Wang C, Feng J: Oxidative stress mediates chemical hypoxia-induced injury and inflammation by activating NF-kappab-COX-2 pathway in HaCaT cells. Mol Cells 2011;31:531-538.

-30 Wang R: Physiological implications of hydrogen sulfide: a whiff exploration that blossomed. Physiol Rev 2012;92:791-896.

-31 Stipanuk MH: Sulfur amino acid metabolism: pathways for production and removal of homocysteine and cysteine. Annu Rev Nutr 2004;24:539-577.

32 Green K, Brand MD, Murphy MP: Prevention of mitochondrial oxidative damage as a therapeutic strategy in diabetes. Diabetes 2004;53:S110-118.

-33 Ray S, Dutta S, Halder J, Ray M: Inhibition of electron flow through complex I of the mitochondrial respiratory chain of Ehrlich ascites carcinoma cells by methylglyoxal. Biochem J 1994;303:69-72.

-34 Chan CM, Huang DY, Huang YP, Hsu SH, Kang LY, Shen CM, Lin WW: Methylglyoxal induces cell death through endoplasmic reticulum stress-associated ROS production and mitochondrial dysfunction. J Cell Mol Med 2016;20:1749-1760.

- 35 Wang P, Xing Y, Chen C, Chen Z, Qian Z: Advanced glycation end-product (AGE) induces apoptosis in human retinal ARPE-19 cells via promoting mitochondrial dysfunction and activating the Fas-FasL signaling. Biosci Biotechnol Biochem 2016;80:250-256.

-36 Nelson MB, Swensen AC, Winden DR, Bodine JS, Bikman BT, Reynolds PR: Cardiomyocyte mitochondrial respiration is reduced by receptor for advanced glycation end-product signaling in a ceramide-dependent manner. Am J Physiol Heart Circ Physiol 2015;309:H63-69.

37 Lan CC, Wu CS, Huang SM, Wu IH, Chen GS: High-glucose environment enhanced oxidative stress and increased interleukin-8 secretion from keratinocytes: new insights into impaired diabetic wound healing. Diabetes 2013;62:2530-2538.

- 38 Usui ML, Mansbridge JN, Carter WG, Fujita M, Olerud JE: Keratinocyte migration, proliferation, and differentiation in chronic ulcers from patients with diabetes and normal wounds. J Histochem Cytochem 2008;56:687-696.

-39 Merkulova Y, Shen Y, Parkinson LG, Raithatha SA, Zhao H, Westendorf K, Sharma M, Bleackley RC, Granville DJ: Granzyme B inhibits keratinocyte migration by disrupting epidermal growth factor receptor (EGFR)mediated signaling. Biol Chem 2016;397:883-895.

40 Liu Z, Xu Y, Chen L, Xie J, Tang J, Zhao J, Shu B, Qi S, Chen J, Liang G, Luo G, Wu J, He W, Liu X: Dendritic epidermal T cells facilitate wound healing in diabetic mice. Am J Transl Res 2016;8:2375-2384.

-41 Tikellis C, Pickering RJ, Tsorotes D, Huet O, Cooper ME, Jandeleit-Dahm K, Thomas MC: Dicarbonyl stress in the absence of hyperglycemia increases endothelial inflammation and atherogenesis similar to that observed in diabetes. Diabetes 2014;63:3915-3925. 